\title{
REVIEW OF IPSS QUESTIONNAIRE IN POSTOPERATIVE TRANSURETHRAL RESECTION OF PROSTATE (TURP) FOR STREAMLINING FOLLOW-UP PROTOCOLS
}

\author{
Graham Broadley ${ }^{1}$, George Delves ${ }^{2}$, Sikander Khwaja ${ }^{3}$ \\ ${ }^{1}$ Urology Registrar. Belvedere Centre, Queens Hospital, Burton - upon - Trent. Burton and Derby \\ University Hospitals NHS Trust. UK. \\ ${ }^{2}$ Urology Consultant. Belvedere Centre, Queens Hospital, Burton - upon - Trent. Burton and Derby \\ University Hospitals NHS Trust. UK. \\ ${ }^{3}$ Urology Consultant. Belvedere Centre, Queens Hospital, Burton - upon - Trent. Burton and Derby \\ University Hospitals NHS Trust. UK
}

Corresponding Author: Broadley.graham@gmail.com

Submitted: February 7, 2019. Accepted: April 11, 2019. Published: May 7, 2019.

\begin{abstract}
\section{Objective}

Clarify the role of IPSS questionnaire for post TURP operation patients, to assess and streamline best follow-up protocols

\section{Materials and Methods}

We identified 87 consecutive patients over 6 months undergoing standardized bipolar TURP. We retrospectively reviewed patients at 3 months in follow-up clinic, where we performed tests including Qmax, Post-void residual (PVR) and IPSS (International Prostate Symptom Score). We identified patients who were discharged or underwent a change in standard management at this point, and used ROC (Receiver Operating Curve) curve analysis to identify the tools which showed the best ability to predict this decision.

\section{Results}

ROC curve analysis suggested Qmax (AUC: 0.7751) and IPSS (AUC 0.8571) were the best tools to predict a change in management. Given the IPSS tool is a questionnaire, thus holding most promise to streamline protocols, we applied Youden-J test to show IPSS=8 cut-off was best to identify management changes.

\section{Conclusion}

The IPSS tool is able to predict a need for change in management in post TURP patients at 3 months. This will allow a simple triage system to provide an efficient and effective decision-making process for discharge without the need for clinic attendance.
\end{abstract}

Keywords: transurethral resection of the prostate, benign prostatic hypertrophy, elective follow-up

Male lower urinary tract symptoms (LUTS) become more common as men age, having both a social and economic impact. The majority of elderly men have at least one symptom. ${ }^{1}$ LUTS have traditionally been labelled as either storage or voiding depending on their profile, with voiding LUTS in men commonly ascribed to bladder outflow obstruction (BOO) secondary to benign prostate enlargement (BPE). 
Currently, male LUTS management options include conservative measures with lifestyle modification and medication, and secondarily surgery. ${ }^{2}$ Surgical options are considered when symptoms are bothersome and impact upon Quality-of-Life (QoL); often when conservative / medical management has failed, or when patients have a permanent urethral catheter and wish to become catheter free.

There is a range of surgical options available in modern urology practice; including Transurethral resection of the prostate (TURP), Transurethral incision of the prostate (TUIP), Holmium laser enucleation of the prostate (HOLEP), Green light laser prostatectomy (GLL), and Urolift. Currently, TURP is the most studied and remains the gold standard procedure; with substantial improvements in post-operation maximum urinary flow rate (Qmax), Post-void residual volume (PVR) and International prostate symptom score (IPSS), with improvements lasting up to 22 years. ${ }^{3}$ TURP has migrated to bipolar technology (TURIS) in recent times as outcomes are similar to monopolar but has an improved safety profile. ${ }^{4}$

EAU guidelines suggest postoperative follow-up should occur between 4-6 weeks with a flow rate, PVR and IPSS, at which point if there are no concerns the patient is discharged from ongoing follow-up. ${ }^{2}$ However, the evidence stated is level 3-4 and recommendation grade $\mathrm{C}$; suggestive of theoretical considerations only without a strong evidence base. This lack of clarity has led to a variety of practice; with follow-up often between 4 weeks and 6 months, in either consultant or nurse led clinics. This study aims to provide some clarity with assessment of local practice at a district general hospital, and shed light onto the best diagnostic tool in follow-up practice.

\section{METHODS}

A total of 87 patients were identified through a theatre tracking database over a 6-month period and patient information collected from electronic patient database (V6 Meditech). This data was then processed and stored on encrypted NHS computers. Data collection parameters included patient demographics, pre and postoperative (3 months) IPSS, Qmax, PVR and relevant change in patient management plans or decision for discharge. "Change in patient management" was defined as: any patient that required further investigation or treatments for LUTS at the 3-month clinic. This included medical and surgical management. All patients that did not have a change in management were discharged from follow-up.

All TURP operations underwent pre-operative assessment, were standardized as bipolar resection (TURIS - plasma vaporization) and underwent routine postoperative management; including inpatient TWOC attempt, and had routine nurse led follow-up at 3 months. Pre-operative catheters did not alter management. The investigations routinely performed at this clinic were PVR, Qmax and IPSS. These tests were performed by the patient with direct instruction / assistance from Urology clinical nurse specialists (CNS). Decisions of further care were Urology CNS led with consultant discussion if required.

Eleven patients were removed from the study because of complete lack of required data; notably patients having unrelated health management which prevented appropriate follow-up, moved away from local region or patient death (unrelated to prostate surgery; one patient). From 76 patients, a total of 50 patients $(65 \%)$ attended our 3-months clinic with 14 patients requiring a change in management for reasons including:

- Prescribed Anti-muscarinics

- Required Bladder neck incision (BNI) / urethrotomy for secondary stricture

- Assessments via Urodynamics -> requires Intermittent self catheterization (ISC) or LongTerm Catheter

- Referral to physiotherapy for pelvic floor exercises Patient demographics are outlined in Table 1.

\section{RESULTS}

Not all patients had a full retrospective data set on electronic record of both pre and postoperative IPSS, Qmax, and PVR; making pre and postoperative change unhelpful within this study. We performed ROC curve analysis of the grouped data for investigation sensitivity / specificity analysis in this 3-month follow-up clinic to include Qmax, PVR and IPSS. We performed Youden J test analysis to determine the optimal IPSS 'cut-off' point to determine management concern. As 
TABLE 1 Patient Demographics

\begin{tabular}{|c|c|c|c|}
\hline $\begin{array}{c}\text { Age } \\
\text { Ave. (range) }\end{array}$ & $\begin{array}{c}\text { ASA } \\
\text { Ave. (range) }\end{array}$ & $\begin{array}{c}\text { Grams Resected } \\
\text { Ave. (range) }\end{array}$ & Pre-operative Catheter \\
\hline $71.6(56-89)$ & $2.6(1-4)$ & $20.4(4-61)$ & 34 \\
\hline
\end{tabular}

TABLE 2 Raw Accumulated Data

\begin{tabular}{|l|c|c|c||c|c|c|}
\hline $\begin{array}{c}\text { Change in } \\
\text { management } \\
\text { plan }\end{array}$ & \multicolumn{3}{|c|}{ No } & \multicolumn{3}{|c|}{ Yes } \\
\hline \multicolumn{1}{|c|}{} & $\begin{array}{c}\text { Qmax } \\
(\mathbf{n}=\mathbf{3 3})\end{array}$ & $\begin{array}{c}\text { PVR } \\
(\mathbf{n}=\mathbf{3 4})\end{array}$ & $\begin{array}{c}\text { IPSS } \\
(\mathbf{n}=\mathbf{3 5})\end{array}$ & $\begin{array}{c}\text { Qmax } \\
(\mathbf{n}=\mathbf{1 0})\end{array}$ & $\begin{array}{c}\text { PVR } \\
(\mathbf{n}=\mathbf{1 2})\end{array}$ & $\begin{array}{c}\text { IPSS } \\
(\mathbf{n}=\mathbf{1 0})\end{array}$ \\
\hline Average & 19.7 & 76.3 & 5.8 & 11.6 & 170.6 & 15.9 \\
\hline Median & 19 & 58 & 4 & 8.3 & 65 & 14 \\
\hline Range & $4.9-42.7$ & $0-240$ & $1-16$ & $5.8-25$ & $0-611$ & $10-25$ \\
\hline
\end{tabular}

a diagnostic test there is difficulty in assessing 'best cut-off' because the test is a compromise between how many it selects versus how many you are prepared to miss, this mathematical tool uses ROC curve sensitivity / specificity values to provide a single statistic to estimate the probability of a correct informed decision. The data accumulated is detailed in Table 2.

\section{ROC CURVES AND IPSS CUT-OFF}

Using the ROC curves, the Youden-J test for IPSS 'best cut-off' showed best probability score of 0.67 when IPSS $=8$ (Figures $1-3$ ).

\section{DISCUSSION / CONCLUSION}

The aim of the project was to clarify and establish local protocols of TURP follow-up patients, as the majority of our patients were discharged and happy at our 3-month follow-up clinic. The EAU guidelines use level 3-4, recommendation grade $C$ evidence for their decision: All patients should have a follow-up clinic to include IPSS, PVR, and Qmax. This required patients to attend for up to 3 hours where patients drink water to facilitate urinary flow assessment. During this time patients would also undergo IPSS discussion with the CNS team. We wanted to attempt to adapt our protocols to reduce both patient waiting time and CNS commitments with associated lost time whilst waiting.

ROC Curve data has suggested that both flow rate $(\mathrm{AUC}=0.7751)$ and IPSS $(\mathrm{AUC}=0.8571)$ showed strong ability to predict the patient outcome for needing a change in management. Clearly, with our objective to streamline the follow-up process, IPSS showed great promise as the best single test to perform with the patient, as this is a 15-minute conversation instead of waiting hours for passing urine. It also had the benefit of assessing patient Quality of life and happiness with LUTS, which is one of the main drivers for patient referral to operation in the first place. This gives an insight into the concept that symptoms of patients are a better tool than objective medical measurements, which is a shift throughout medicine with patient-reported outcome measures (PROMS ${ }^{5}$ ) becoming more prominent. PVR seems to have no predictive potential $(\mathrm{AUC}=0.5585)$, with some patients having up to $240 \mathrm{~mL}$ as residual but remain happy, and required no further medical input.

Assessment of IPSS cut-off for continued medical concern we used Youden-J Test, as this test incorporates sensitivity and specificity generated by ROC analysis, and results in the likelihood a patient with a positive result will have a complication. We determined the 
FIG. 1 Postoperative Qmax predicting change in management.

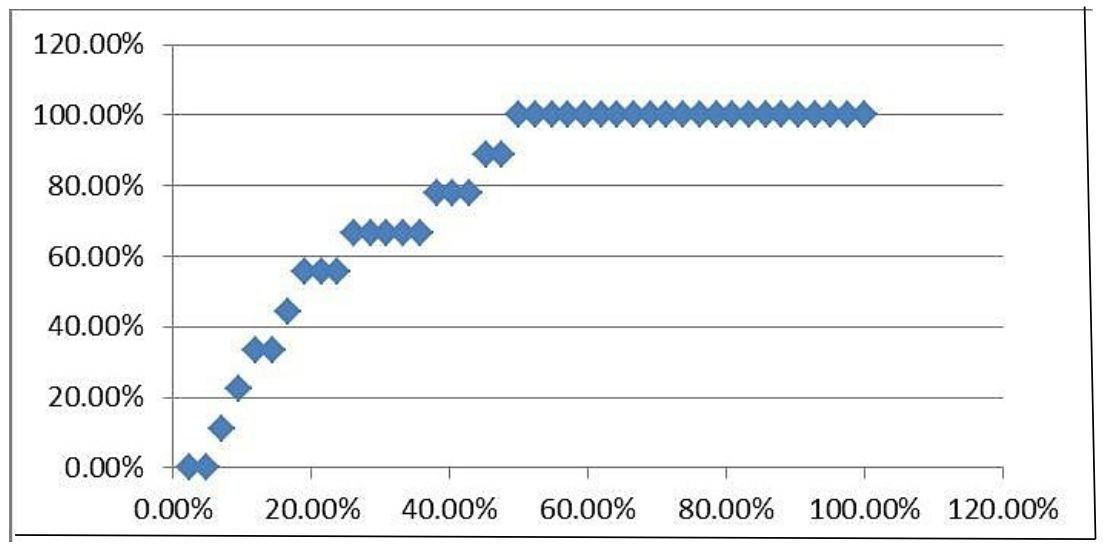

AUC (Area Under Curve): 0.7751

FIG. 2 Postoperative PVR predicting change in management.

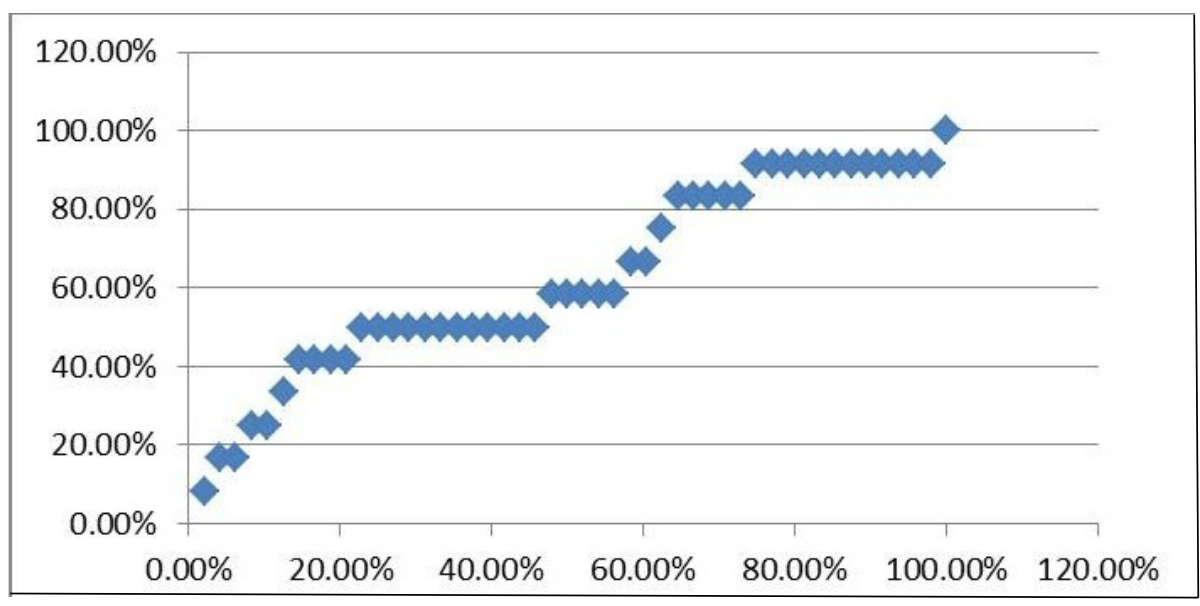

AUC: 0.5585

best placed would be IPSS $=8$ (Youden-J probability $=0.67$ ). As such, patients with IPSS score 7 and lower would be safe for discharge, and patients with score 8 or greater would require further investigations namely Qmax and PVR. This would have the potential to 'triage' patients with a need to a change in their management or to be discharged. The IPSS score of 8 has symmetry for the IPSS tools cut-off for moderate LUTS is 8 also.

This department feels with application of an IPSS triage service prior to flow clinic assessment would reduce nursing urine flow clinic commitments allowing reallocation of resource to other under-pressure services. Patient benefits would allow reduced clinic time commitments, reducing parking charges and travel difficulties given patients are often elderly and do not drive. The potential final conclusion for the future, would be the triage clinic could be a telephone consultation instead reducing issues further.

We had 87 consecutive patients identified as undergoing TURP in a 6-month window with only 11 removed from data set. Of these, $65 \%$ attended our 3 -month postoperative clinic with vast majority having full data set in the postoperative setting. Unfortunately, 
FIG. 3 Postoperative IPSS predicting change in management.

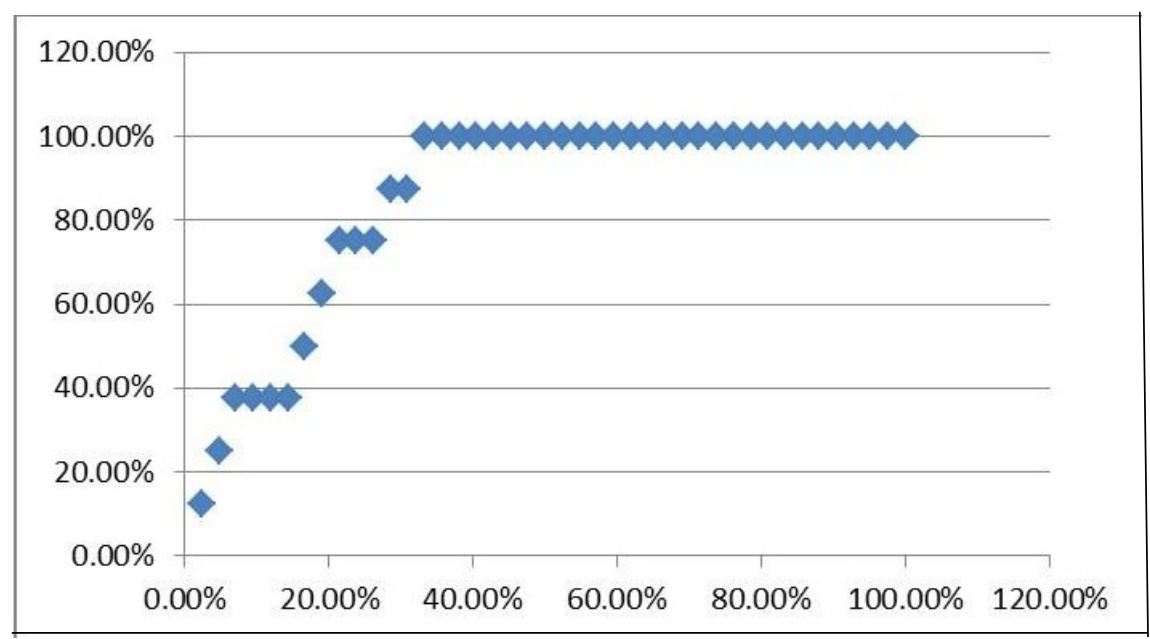

AUC: 0.8571

retrospective documentation of pre-operative data was less complete in interrogated electronic records and prevented a direct pre and postoperative comparison. Whilst this may have improved our data set it was always our intention to concentrate on the postoperative setting thus impact on our validity is not compromised. We believe this study is representative of patient outcomes and applicable to a wider context nationally. We had a postoperative requirement for: urethrotomy rate $2.6 \%$, ISC rate $3.9 \%$ and anti-muscarinic prescription rate $5.2 \%$.

Overall, this study provides a review of common postoperative TURP practice which has shown the IPSS as the best tool to determine outcome and progress at follow-up, and may show systemic improvements for both the NHS and patients progress through their care.

\section{REFERENCES}

1. Gravas S EAU 2015. Societe Internationale d'urologie (SIU). Lower urinary tract symptoms (LUTS): an international consultation on male LUTS. C Chapple and P Abrams, editors; 2013. Available at: http://www
.siu-urology.org/themes/web/assets/files/ICUD/pdf/ Male\%20Lower\%20Urinary\%20Tract\%20Symptoms\%20(LUTS).pdf. Accessed via EAU guidelines of non-neurogenic-male-LUTs accessed 14 May 2018.

2. Gravas $S$ et al EAU update march 2015. Management of Non-Neurogenic Male Lower Urinary Tract Symptoms (LUTS). EAU Guidelines. Available at: https://uroweb.org/ wp-content/uploads/EAU-Guidelines-Non-NeurogenicMale-LUTS-Guidelines-2015-v2.pdf.

3. Reich $\mathrm{O}$, et al. Techniques and long-term results of surgical procedures for BPH. Eur Urol 2006 Jun;49(6):970-8; discussion 978. Epub 2006 Feb6. Available at: https:// www.ncbi.nlm.nih.gov/pubmed/16481092.

4. Omar et al MI. Systematic review and meta-analysis of the clinical effectiveness of bipolar compared with monopolar transurethral resection of the prostate (TURP). BJU Int 2014 Jan;113(1):24-35. doi: 10.1111/ bju.12281. Epub 2013 Oct 24. https://www.ncbi.nlm .nih.gov/pubmed/24053602.

5. Devlin NJ. 'Getting the most out of PROMS'. The Kings Fund. Available at: https://www.kingsfund.org.uk/sites/ default/files/Getting-the-most-out-of-PROMs-NancyDevlin-John-Appleby-Kings-Fund-March-2010.pdf. 